\title{
Markov random field based English Part-Of-Speech tagging system
}

\author{
Sung-Young Jung, Young C. Park, Key-Sun Choi and Youngwhan Kim* \\ Computer Science Department \\ Korea Advanced Institute of Science and Technology \\ Taejon, Korea \\ * Multimedia Research Laboratories \\ Korea Telecom \\ \{chopin,ycpark,kschoi\}@essone.kaist.ac.kr
}

\begin{abstract}
Probabilistic models have been widely used for natural language processing. Part-of-specch tagging, which assigns the most likely tag to each word in a given sentence, is one of the problems which can be solved by statistical approach. Many researchers have tried to solve the problem by hidden Markov model (HMM), which is well known as one of the statistical models. But it has many difficulties: integrating heterogeneous information, coping with data sparseness problem, and adapting to now environments. In this paper, we propose a Markov radom field (MRF) model based approach to the tagging problem. The MRF provides the base frame to combine various statistical information with maximum entropy (MF) method. As Gibbs distribution can be used to describe a posteriori probability of tagging, we use it in maximum a posteriori (MAP) estimation of optimizing process. Besides, several tagging models are developed to show the effect of adding information. Experimental results show that the performance of the tagger gets improved as we add more statistical information, and that MRE-based tagging model is better than HMM based tagging model in data sparseness problem.
\end{abstract}

\section{$1 \quad$ Introduction}

Part-of-speech tagging is to assign the correct tag to each word in the context of the sentence. 'There are three main approaches in tagging problem: rule-based approach (Klein and Simmons 1963; Brodda 1982; Paulussen and Martin 1992; Brill et al. 1990), statistical approach (Church 1988; Merialdo 1994; Foster 1991; Weischedel et al. 1993; Kupiec 1992) and connectionist approach (Bencllo et al. 1989; Nakamura et al. 1989). In these approaches, statistical approach has the fol- lowing advantages:

- a theoretical framework is provided

- automatic lcarning facility is provided

- the probabilities provide a straightforward way to disambiguate

Many information sources must be combined to solve tagging problem with statistical approach. It is a significant assumption that the correct tag can generally be chosen from the local context. Not only local sequences of words and tags are needed to solve tagging problem, but syntax, semantic and morphological level information is also required in general. Usually information sources such as bigram, trigram and unigram are used in the tagging systems which are based on statistical method. 'Traditionally, linear interpolation and its variants have been used to combine the information sources, but these are shown to be seriously deficient.

ME (Maximum Entropy) estimation method provides the facility to combine sevoral information sources. Fach information source gives rise to a set of constraints, to be imposed on the conlbined estimate. The function with the highest entropy within the constraints is the ME solution. Given consistent statistical evidence, a mique $\mathrm{MF}$ solution is guaranteed to exist and an iterative algorithm is provided.

MRE (Markov random field) model is based on ME method and it has the facility to combine many information sources through feature functions. MRF model has the following advantages: robustness, adaptability, parallelism and the facility of combining information sources. MRF-based tagging model inherits these advantages.

In this paper, we will present one of the statistical models, namely MRF-based tagging system. We will show that several information sources including unigram, bigram and trigram, can be combined in MRF-based tagging model. Experimental results show that the MRF-based tagger has very good performance especially when training data size is small.

Section 2 describes the tagging problem, Section 3 describes statistical model already known 
and section 1 the research for combining statistical information. Section 5 provides MRl-based tagging model and section showes the experimontal results. Section 10 compares MRF with II MM. Finally we conclude in section II.

\section{The Problem of Tagging}

When sentence $W=w_{1}, w_{2}, \ldots, w_{n}$ is given, there cxist corresponding tags ' $T$ ' $=t_{1}, t_{2}, \ldots, t_{n}$ of the same length. We call the pair (W, l') an alignmont. Wo say that word $w_{i}$ has been assigned the tag $t_{i}$ in this aligmment. Wo suppose that a set of latgs is given. 'Tageging is assigning corrod tag seguenee ' $t=t_{1}, t_{2}, \ldots, t_{n}$ for given word secpuence $W=w_{1}, w_{2}, \ldots, w_{n}$

\section{Probabilistic Formulation(HMM)}

lot us assume that we want to know the most likely tag secuunee $\phi(W)$, given a particular word secuence $W$. The tageging problem is defined as linding the most likely tage sequence ' $?$ '

$$
\begin{aligned}
\phi(W) & =\arg \max _{T^{\prime}} l^{\prime}\left(T^{\prime} \mid W\right) \\
& =\arg \max _{T^{\prime}} \frac{P\left(\left.W\right|^{\prime}\right) l^{\prime}\left(I^{\prime}\right)}{P^{\prime}(W)} \\
& =\arg \max _{T} P\left(|W|^{\prime}\right) P(I)
\end{aligned}
$$

where $P(T)$ is the a priori probability of a tag sequence ' $\mathrm{T}$, $P(W \mid T)$ is the conditional probability of word sequence $W$, given the secfurnee of tags $' T$, and $P(W)$ is the anconditioned probability of word sequence $W$. 'The probability $P^{\prime}(W)$ in $(2)$ is removed because it has no eflect on $\phi(W)$. Consequently, it is sufficient to find the tag secpuence 'T' which salisfies (3).

We can rewrite the probability of each sequence as a product of the conditional probabilitios of ach word or tag given all of the previous tags.

$$
\begin{aligned}
& P\left(W \mid T^{\prime}\right) P^{P}(T) \\
& =I_{i=1}^{n}
\end{aligned} \quad\left\{\begin{array}{l}
P^{\prime}\left(w_{i} \mid l_{i}, \ldots, l_{1}, w_{i-1}, \ldots, w_{1}\right) \\
\times P\left(l_{i} \mid l_{i-1}, \ldots, l_{1}\right)
\end{array}\right\}
$$

'Typically, one makes two simplifying assumptions to cut down on the number of probabilities to be ostimated. First, rather than assuming $w_{i}$ depends on all previous words and all previous tags, one assumes $w_{i}$ depends only on $l_{i}$. Second, rather than assuming the tig $t_{i}$ depends on the full sequence of previous tags, we can assume that local context is sullicient. 'This locality assumed is refered to as a Markov independence assumption.

Using these assumption, we approxinnate the equation to the following

$$
\begin{aligned}
P^{P}\left(W \mid T^{\prime}\right) & \simeq \mathbb{I L}_{i=1}^{n} P^{\prime}\left(w_{i} \mid l_{i}\right) \\
P(T) & \simeq \mathbb{I}_{i=1}^{n} P\left(t_{i} \mid l_{i-1}\right)
\end{aligned}
$$

Accordingly, $\phi(W)$ is derived by applying (A) and (5) to (3).

$$
\phi(W)=\arg \max _{T} I_{i=1}^{n} P\left(w_{i} \mid t_{i}\right) P\left(t_{i} \mid t_{i-1}\right)
$$

We can get rach probability value from the tagged corpus which is prepared for training by using ( $(7)$ and (8).

$$
\begin{aligned}
P\left(w_{i} \mid t_{i}\right) & =\frac{C\left(w_{i}, t_{i}\right)}{C\left(t_{i}\right)} \\
P\left(t_{i} \mid t_{i-1}\right) & =\frac{C\left(t_{i-1}, t_{i}\right)}{C\left(t_{i}\right)}
\end{aligned}
$$

where $C\left(t_{i}\right), C\left(t_{i}, t_{j}\right)$ is the frequency obtained from traning data.

Viterbi algorithm (Forney 73) is the one gene?ally used to lind the tag secuener which safisfies (6) and this algorithm guarantes the optimal solution to the problem.

'This model has several problems. liest, some? words or tag secpuences may not occur in training clatia or may occur with very low frequency; nevertheless, the words or lag sequences can aptpear in tagging process. In this case, it usually causes very had result to compute (6). because the probability has zero value or very low value. 'This problem is called data sparseness problem. 'To avoid this problem, smoothing of information must be used. Smoothing process is almost essential in IIMM because IIMM has severe data sparseness problem.

\section{4 combining information sources}

\section{1 linear interpolation}

Various kinds of informalion soures and different knowledge sourees must be combined to solve the lagging problem. The general method nsed in IIMM is linear interpolation, which is the weighted summation of all probalsility information sources.

$$
P_{\text {rombined }}(w \mid h)=\sum_{i=1}^{k} \lambda_{i} P_{i}(w \mid h)
$$

where: $0<\lambda_{i} \leq 1$ and $\sum_{i} \lambda_{i}=1$.

This method can be used both as a way of combining knowledge sources and smoothing information sources.

II MM based tagging model uses unigram, bigratu and trigram information. 'These information somress are lincarly combined by weighted summa1.ion. 
$\tilde{P}\left(t_{i} \mid t_{i-1}, t_{i-2}\right)=\lambda_{1} P\left(t_{i} \mid t_{i-1}, t_{i-2}\right)+\lambda_{2} P\left(t_{i} \mid t_{i-1}\right)$

where $\lambda_{1}+\lambda_{2}=1$. The parameter $\lambda_{1}$ and $\lambda_{2}$ can be estimated by forward-backward algorithm (Deroua86+) (Charniak93+) (HUANG90+).

Linear interpolation is so advantageous because it reconciles the different information sources in a straightforward and simple-minded way. But such simpliticy is also the source of its weaknesses:

- Linearly interpolated information is generally inconsistent with their information sources because information sources are heterogeneous for each other in general.

- Iincar interpolation does not make optimal combination of information sources.

- Linear interpolation has over-estimation problem because it adjusts the model on the training data only and has no policy for untrained data. This problem occur seriously when the size of the training data is not large enough.

\subsection{ME(maximum entropy) principle}

There is very powerful estimation method which combines information sources objectively. $\mathrm{ME}$ (maximum cntropy) principle (Jaynes57) provides the method to combine information sources consistently and the ability to overcome overestimation problem by maximizing entropy of the domain with which the training data do not provide information.

Let us describe ME principle briefly. For given $x$, the quantity $x$ is capable of assuming the discrete values $x_{i},(i=1,2, \ldots, n)$. We are not given the corresponding probabilities $p_{i}$; all we know is the expectation value of the function $f_{r}(x),(r=$ $1,2, \ldots, m)$ :

$$
E\left[f_{r}(x)\right]=\sum_{i=1}^{n} p_{i}\left(x_{i}\right) f_{r}\left(x_{i}\right)
$$

On the basis of this information, how can we determine the probability value of the function $p_{i}(x)$ ? At first glance, the problem seems insoluble because the given information is insufficient to determine the probabilities $p_{i}(x)$.

We call the function $f_{r}\left(x_{i}\right)$ a constraint function or feature. Given consistent constraints, a unique ME soluton is guaranteed to exist and to be of the form:

$$
p_{i}\left(x_{i}\right)=e^{-\sum_{r} \lambda_{r} f_{r}\left(x_{i}\right)}
$$

where the $\lambda_{r}$ 's are some unknown constants to be found. 'This formula is derived by maximizing the entropy of the probability distribution $p_{i}$ as satisfying all the constraint given. To search the $\lambda_{r}$ 's that make $p_{i}(x)$ satisfy all the constraints, an external

observation:

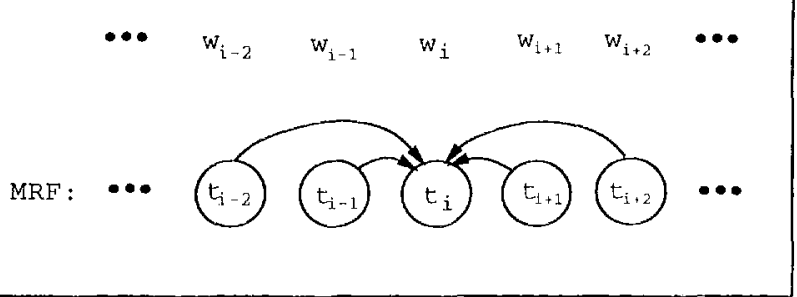

Figure 1: $M R F^{\prime} \mathrm{T}$ ' is defined for the neighborhood system with distance 2

iterative algorithm, "Generalized Iterative Scaling" (GIS), exists, which is guaranteed to converge to the solution (Darroch72+).

(12) is similar to Gibbs distribution, which is the primary probability distribution of $\mathrm{MRF}$ model. MRF model uses ME principle in combining information sources and parameter estimation. We will describe MRF model and its parameter estimation method later.

\section{$5 \quad$ MRF-based tagging model}

\subsection{MRF in tagging}

Neighborhood of given random variable is defined by the set of random variables that directly affect the given random variable. Let $N(i)$ denote a set of random variables which are neighbors of $i$ th random variable. Let's define the neighborhood system with distance $L$ in tagging for words $W=$ $w_{1}, \ldots, w_{n}$, and tags $T=t_{1}, \ldots, t_{n}$.

$$
N(i)=\{i-L, \ldots, i-1, i+1, \ldots, i+L\}
$$

This neighborhood system has one dimensional relation and describes the one dimenstional structure of sentence. Fig. I showes MRF $T$ which is defined for the neighborhood system with distance 2. The arrows represent that the random variable $t_{i}$ is affected by the neighbors $t_{i-2}, t_{i-1}, t_{i+1}, t_{i+2}$. It also showes that $t_{i}, t_{i-1}$ and $t_{i}, t_{i+1}$ have the neighborhood relation connected by bigram, and that $t_{i}, t_{i-1}, t_{i-2}$ and $t_{i}, t_{i+1}, t_{i+2}$ have the neighborhood relation connected by trigram.

A clique is defined as the set of random variables that all of the pairs of random variables are neighborhood in it. Let's define the clique as the tag sequence with size $L$ in tagging problem.

$$
C_{i}=\left\{t_{i-L}, t_{i-\left(L_{-}-1\right)}, \ldots, t_{i}\right\}
$$

A clique concept is used to define clique function that evaluates current state of random variables in clique.

The definition of MRF is presented as following.

Definition of MRF: Random variable $T$ is Markov random field if $T$ satisfies the following two properties. 
Positivity:

$$
P(T)>0, \forall I^{\prime}
$$

Locality :

$$
P\left(t_{i} \mid t_{j}, \forall j, j \neq i\right)=P\left(t_{i} \mid t_{j}, \forall j, j \in N(j)\right)
$$

We assume that every probablity value of tag sequence is larger than zero because ungrammatical sentences can appear in human language usage, including meaningless sequence of characters. So the positivity of MRF is satisfied. This assumption results in the robustness and adaptability of the model, even though untrained events occur.

'T'he locality of MRR is consistent with the assumption of tagging problem in that the tag of given word can be detemined hy the local context. Consequently, the random variable $T$ is MRF for neighborhood system $N(i)$ as 'T' satisfies the positivity and the locality.

\subsection{A Posteriori Probability}

A posteriori probability is needed to search for the most likely tag sequonce. MRI provides the theoretical background about the probability of the system (Besag74) (Goman84t).

IIammersley-Clifford theorem: The probability distribulion $P(T)$ is Gibbs distribution if and only if random variable ' $T$ ' is Markov random firld for given neighorhood system $N(i)$,

$$
\begin{aligned}
P(T) & =\frac{e^{-\frac{1}{T^{2}+n} U(T)}}{Z} \\
Z & =\sum_{T^{\prime}} e^{-\frac{1}{T^{\prime} r} U(T)}
\end{aligned}
$$

where ' $T$ ' $m$ is temperature, $\%$ is normalizing constant, called partition function and $U\left({ }^{\prime} I\right)$ is energy function. 'The a priori probability $P(T$ ) of tag sequence ' $T$ is Gibbs distribution because the random variable ' $T$ ' of tagging' is MRL'.

It can be proved that a posteriori probability $P(T \mid W)$ for given word soquence $W$ is also Cubbs distribution (Chun93). Consequently, a posteriori probability of ' $I$ ' for given $W$ is

$$
P(T \mid W)=\frac{1}{Z^{\prime}} e^{-\frac{1}{T^{\prime}+2} U(T \mid W)}
$$

We use (19) to carry ont MAP'estimation in the tagging model. 'Tho energy function $U(I \mid W)$ is of this form.

$$
U\left(I^{\prime} \mid W\right)=\sum_{c} V_{c}\left(I^{\prime} \mid W\right)
$$

where $V_{c}$ is elique function with the property that $V_{c}$ depends only on those random variable in clique $c$. 'This means that energy finction can be obtained from each clique funtion which splits the set of random variables to subsets.

\section{Clique function design}

The more state of random variables are near to the solution, the more the system becomes stable, and cnergy function has lower value. Fnergy function represents the degree of unstability of current state of random variables in MRF. It is similar to the behaviour of molecular particles in the real world.

Clique function is proportional to energy function, and it represents the unstability of current state of random variables in clique or it has high value when the state of MRF is bad, low value when the state of MRI' is near to solution. Clique function contribules to reduce the computation of cvaluation function of entire MRF' by clique concept that separates random variables to the subsets.

Clique function $V_{i}\left(T^{\prime} \mid W\right)$ is described by the fon tures that represent the constraint or information sources of given problem domain.

$$
V_{i}(T \mid W)=\sum_{r} \lambda_{r} f_{r}^{i}(T \mid W)
$$

\subsection{MIR.F Model 1 (Basic model)}

The basic information sources which are used in statistical tagging model are unigram, bigram and trigram. MRE model 1 uses unigram, bigram and trigram. We write the feature function of unigram ass

$$
\int_{\text {unigram }}^{i}==\left(1-P\left(t_{i} \mid w_{i}\right)\right)
$$

and the feature function of n-gram, including bigram, trigram as

$$
\int_{n-q r a m}^{i}=\sum_{j \in N(i)}\left(1-I^{\prime}\left(t_{i} \mid j\right)\right)
$$

where

$P\left(t_{i} \mid j\right)= \begin{cases}P\left(t_{i} \mid t_{i-j}, t_{i-j+1}, \ldots, t_{i-1}\right), & \text { if } i>j \\ P\left(t_{i} \mid t_{i+1}, t_{t+2}, \ldots, t_{i+j}\right), & \text { if } i<j\end{cases}$

The clique function of the model 1 is made as follows.

$$
V_{i}\left(I^{\prime} \mid W\right)=\lambda_{1} \cdot f_{u n i g r a m}+\lambda_{2} \cdot f_{n-g r a m}
$$

\subsection{Model 2 (Morphological information included)}

Morphological level information helps tagger to detcrmine the tag of the word, more especially of the unknown word. 'The sutfix of a word gives very useful information about the tag of the word in Finglish. 'The clique function of model 2 is defined as

$$
f_{s u f f i x}^{i}=\left(1-P\left(t_{i} \mid s u f f i x\left(w_{i}\right)\right)\right)
$$

We used the statistical distribution of the sixty suffixes that are most frequently used in linglish. 
We can expand the clique function of the model 1 easily by just adding suffix information to the clique function of the model 2.

$$
V_{i}(T \mid W)=\lambda_{1} \cdot f_{\text {unigram }}+\lambda_{2} \cdot f_{n-g r a m}+\lambda_{3} \cdot f_{\text {suffix }}
$$

\subsection{Model 3 (error correction)}

There exist error prone words in every tagging system. We adjust error prone words by collecting the error results and adding more information of the words. The feature function of Model 3 is for adjusting errors in word level.

$$
\begin{array}{r}
f_{\text {error } 1}^{i}=\left(1-P\left(t_{i} \mid t_{i-1}, w_{i}, t_{i+1}\right)\right) \\
f_{\text {error } 2}^{i}=\left(1-P\left(t_{i} \mid w_{i-2}, t_{i-1}\right)\right)
\end{array}
$$

We used the probability distribution of five hundred error prone words in Model 2 in order to reduce the number of parameters.

\section{Optimization}

The process of selecting the best tag sequence is called as optimization process. Wo use $\mathrm{MAP}$ (Maximum A Posteriori) estimation method. 'The tag scquence $T$ is selected to maximize the a posteriori probability of tagging (19) by $\mathrm{MAl}$.

Simulated annealing is used to search the optimal tag sequence as Cibbs distribution provides simulated annealing facility with temperature and energy concept. Wo change the tag candidate of one word selected to minimize the encrgy function in $k$-th step from $T^{(k)}$ to $T^{(k+1)}$, and repeat this process until there is no change. The temperature' $I$ ' $m$ is started in high value and lower to zero as the above process is doing. 'I'hen the final tag sequence is the solution. Simulated annealing is useful in the problem which has very huge search space, and it is the approximation of MAP estimation (Geman84t)

There is another algorithm called Vilerbi algorithm to find optimal solution. Viterbi algorithm gnarantees optimal solution but it cannot be used in the problem which has very huge search space. So it is used in the problem which has small search space and used in HMM. MRF model can use both Vitcrbi algorithm and simulated ancaling, but it is not known to use simulated annealing in HMM.

\section{8 parameter estimation}

The weighting parameter $\lambda$ in the clique function (19) can be estimated from traning data by ME principle (Jaynes57).

lot us describe ME principle and IIS algorithm briefly. For given $x=\left(x_{1}, \ldots, x_{n}\right)$, the corresponding probabilities $p_{i}\left(2_{i}\right)$ is not known. All we know is the expectation value of the function $f_{r}(x),(r=1,2, \ldots, m)$ :

$$
E\left[f_{r}(x)\right]=\sum_{i=1}^{n} p_{i}\left(x_{i}\right) f_{r}\left(x_{i}\right)
$$

Given consistent constraints, we can find the probability distribution $p_{i}$ that makes the entropy $-\sum p_{i} \ln p_{i}$ value maximum by using Largrangian multipliers in the usual way, and obtain the result:

$$
p_{i}\left(r_{i}\right)=\exp \left(-\sum_{r} \lambda_{r} f_{r}\left(x_{i}\right)\right)
$$

This formula is almost similar to Gibbs distribution (17), also $f_{r}$ corresponds to the feature of clique function in MRF (20) (21). Using this fact, we can use ML in parameter estimation in MRF.

Wo can derive (31) to be used in parancter estimation from training data.

$$
\begin{aligned}
& -\frac{\partial}{\partial \lambda_{r}} \ln Z=\sum_{i} p_{i} f_{r}\left(x_{i}\right) \\
& Z=\sum_{i} \exp \left(\sum_{r} \lambda_{r} f_{r}\left(x_{i}\right)\right)
\end{aligned}
$$

To solve the solution of it, a mumerical analysis method GIS (Generlaized Iterative Scaling) was suggested (Jarroch72+). Pietra used his own algorithm IIS (Improved Iterative Scaling) based on Gis to induce the features and parameters of random field automatically (Pictra95). Following is IIS algorithm

\section{IIS(Improved Iterative Scaling)}

- Initial data

A reference distribution $\bar{p}$, an initial model $q_{0}$ and $f_{0}, f_{1}, \ldots, f n$.

- Output, $q_{*}$ and $\lambda$ by Mlis estimation

- Algorithm

(0) Set $q^{(0)}=q_{0}$

(1) For each $i$ find $\lambda_{i}$, the mique solution of

$$
\sum_{T} q^{(k)} f_{i}(T) e^{\lambda^{(k)}} \sum_{r} f_{r}(T)=\sum_{T} \tilde{p}(T) f_{i}(T)
$$

(2) $\mathrm{k} \leftarrow \mathrm{k}+1$, set $q^{k+1}$ with new $\lambda_{i}$

(3) If $q^{(k)}$ has converged, set $q_{*}=q^{(k)}$ and terminate. Otherwise go to $\operatorname{stcp}(1)$

where $q^{(k)}$ is the distribution of the model in $\mathrm{k}$ th step, and it corresponds to the posteriori probability of the tagging model (19). $\lambda$, the solution of (33) can be obtained by Newton method (Curtis $89+$ ), one of numerical analysis method.

'The roference distribution $\hat{p}$ is the probability distribution which is obtained directly from training data. $\tilde{y}$ corresponds to the posterior distribution $I^{\prime}\left(T^{\prime} \mid W\right)$ in the tagging model. We use the 


\begin{tabular}{|c|c|}
\hline Model & Tagging accuracy $(\%)$ \\
\hline IIMM & 96.11 \\
MRF(1) & 96.2 \\
MRF(2) & 96.5 \\
MRF(3) & 97.1 \\
\hline
\end{tabular}

Table 1: Neasuring the accuracy of IIMM and MRF models.

posterior probability of the words sequence of win(low size $n$ (especially 3 in this model) by comnting the entry on training data. Training data means tagged corpus here.

$$
\check{p} \simeq P\left(t_{1}, \iota_{2}, \ldots, l_{n} \mid w_{1}, w_{2}, \ldots, w_{n}\right)
$$

\section{$9 \quad$ Experiments}

'The main objective of this experiments is to pare the MRE tagging model with the II YM lakgging model. Wo constructed a MRF lagger and a II MM tagger using same information on the same cnvironment.

It is necessary to do smoothing process for clata sparseness problem which is severe in $I \mathrm{MM}$, while MRF has the facility of smoothing in itself like neural-net. Wo used linear interpolation method (Derona86+) (jolinek89) and assigning frequency I for unknown word (Weisch9.3+) for smoothing in II MM

Wo used the Brown corpus in P'enultree Bank, described in (Marens93+) with 48 different tags. $A$ set of 800,000 words is collected for eacle part of Brown corpus and used as training data, which is used to build the modcls. And a set of 30,000 words corpus is used as test data, which is used to test the quality of the models.

Table I shows the accuracy of each tagging model. The average accuracy of the IIMM-based tagger is similar to that of MlRF(1) tagger because they use the same information.

ligg. 2 shows that the eror rate as the size of training data is increased. MIRE(1) has lower error rate than that of $\mathrm{IIMM}$ when the size of training data is small. "The cror rate of MRF(2) is decreased especially when the size of the trajuing data is stnall, because morphological information helps the process of unknown words. Finally, MRE(3) show improvencent as the size of training data grows but converges to the limit on some points.

These experiments show that MRF' has better addaplabilily with small training datia than IIMM does, and that MRE tageger has less data sparseness problem than II MM tagger.

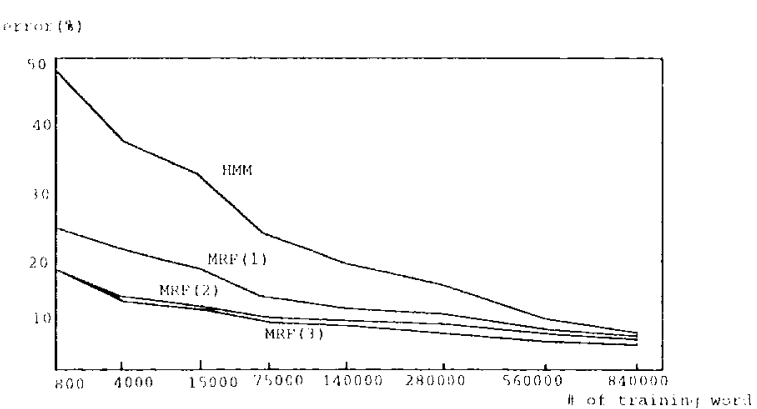

Figure 2: Frros rate of each model for given size of training word

\section{Comparison of MRF with HMM}

We can derive the simplified equation of $11 \mathrm{MM}$ only with bigran!

$$
P^{\prime}\left(T^{\prime} \mid W\right)=I^{\prime}\left(t_{2} \mid t_{1}\right) I^{\prime}\left(t_{3} \mid t_{2}\right) \ldots I^{\prime}\left(t_{n} \mid t_{n-1}\right)
$$

(35) is considered as the multiplied probalsilties of a the local events. The nearer the probability value of local rvent is to zero, the more it alfects the probability of the cotire event. 'This property strictly reflects on the events which does not occur in training data. But it prohibits cven the event. that does not occur in training data, althongh the cvent is legal.

MRI can be simplified by the summation of clique function as (36).

$$
P(T \mid W)=\frac{1}{Z} e^{-\frac{1}{T} \cdot \frac{1}{m}\left\{V_{1}+V_{2}+\ldots+V_{n}\right\}}
$$

MRF uses rvaluation function by summation, while IIMM does by multiplication. Fven if a clique function value is very bad, other clicue function can compensate adequately becanse the clique functions aro connected by summation. 'There is no critical point of posteriori probablity in MRF, while IIMM has critical point in zero value. This property results in the rolustness and the adaptability of the model and makes MRI' molel stronger in data sparseness problem.

\section{Conclusion}

We proposed a MRI-based tagging model. Information sources for tagging are combined by ME principle which is usced in MRF as theoretical background. All parameters used in the model are estimated from training data automatically. As a result, our MRE-based tagging model has bet.ter performance than IIMM tagging model, especially when the size of the training data is small. Wo have seen that the performance of the MRl'based tagging nodel can be improved by adding information to the model. 


\section{References}

Besag, J. "Spatial interaction and the statistical analysis of lattice systems( with discusstion )," J. Royal Statist. Soc., serics B, vol. 36, pp. 192326, 1974.

Besag, J. "On the Statistical Analysis of Dirty Pictures", J. Royal Statist. Soc., vol. B48, 1986.

Brill, E. "A Simple Rule-Based Part of Speech 'lagger", In Proceedings of the 3rd Conf. on Applied Natural Language Processing, pages 153155, April, 1992.

Charniak, E., C. Hendrickson, N. Jacobson and M. Perkowitz, "Equations for Part-of Speech Tagging," Proc. of Nat'l Conf. on Artificial Intelligence( $A$ A A I-86), pp. 784-789, 1993.

In, G. Chun, "Range Image Segmentation Using Multiple Markov Random Fields", Ph.D. thesis, KAIST, KOREA, 1993.

Church, K. W., "A Stochastic PARTS Program and Noun Phrase Parser for Unrestricted Text,", Proceedings of Applied Natural Language Processing, Austin, 'Texas, pp. 136-143, 1988.

Darroch, J. N. and D. Ratcliff, "Generalized Iterative Scaling for Long-Linear Models..", The Annals of Mathematical Statistics, Volume 43, pages 1470-1480, 1972.

Derouault, A. M. and B. Merialdo, "Natural Language Modeling for Phoneme-to-Text Transcription,", IEEE Tr. on Pattern Anaysis and Machine Intelligence, vol. PAMI-8, no.6, Nov. 1986.

Curtis, F. G. and Patric O. Wheatley, "Applied Numerical Analysis", forth edition, ADDISON WESLEY, 1989.

Forney, G. D., "The Viterbi Algorithm", Proc. of the IEEF, vol. 61, pp. 268 278, Mar. 1973.

Gamble, F. B., Geiger D. and Possio 'T., "Integration of Vision Modules and labeling of Surface Discontinuities", IEEE Transactions on systems, man and cybernetics, vol. 19, no. 6, November/decemver 1989.

Geman, S. and Geman D., "Stochastic Relaxation, Gibbs Distributions, and the Bayesian Restoration of Images", IEEE transactions on pattern analysis and machine intelligence, Vol.PAMI-6, NO. 6, NOVEMBER 1984.

Geiger, D. and Girosi F., "Parallel and Deterministic Algorithms from MRF's: Surface Reconstruction", IEEE Transactions on pattern analysis and machine intelligence, VOI, 13, NO. 5, MAY 1991.

IIUANF, X.D., Y. ARIKI and M.A. JACK, "Hidden Markov Models for Speech Recognition", 1990 .
Jaynes, E. 'T., Information Theory and Statistical Mechanics, Physics Reviews.106, pages 620-630, 1957.

Jelinek, F. , "Self-Organized Language Modeling for Speech Recognition.", in Readings in Specch Recognition, Alex Waibel and Kai-Fu Lee(Fditors). Morgan Kaufmann, 1989.

Kupiec, J., Robust Part-of-Speeh 'Tagging Using a IIidden Markov Model, Computer Speech and language, 1992.

Marcus, M. P., Beatrice Santorini and Mary Ann Marcinkiewiez, "Building a large annotated corpus of English: the Penn 'Ireebank", Computational Linguistics, Vol. 19, No. 2, pp 313-330, June, 1993.

Merialdo, B., "Tagging English Text with a Probabilistic Model" , Computational Linguistics, Volume 20, no 2, June 1994.

Nakamura, M., K. Maruyama, T. Kawanata and K. Shikano, "Neural Network Approach of Word Category Prediction for Rnglish Texts," Int'l Conf on Computational linguistics(Coling-90), pp. 213-218, 1990.

Pietra, S. D., V. D. Pietra and J. Lafferty, "Inducing features of random fields", Carnegie Mellon University, Technical report CMU-CS-95-144, MAY, 1995.

Rosenfeld, R., "Adaptive Statistical language Modeling: A Maximum Lntropy Approach" , Carnegie Mellon University,technical report CMU-CS-94-138, April 19, 1994.

Weischedel, R., R. Scewartz, J. Ralmucci, M. Meteer, and L. Rawshaw. "Coping with Ambiguity and Unknown Words through Probabilistic Models", Computational Linguistics, 19(2):359-382, 1993.

Zhang, J. and J.W. Modestino, "A Markov Random Field model-based approach to image interpretation", Visual Communications and image Processing IV, Vol 1199, 1989. 